\title{
Abnormal left-sided orbitomedial prefrontal cortical-amygdala connectivity during happy and fear face processing: a potential neural mechanism of female MDD
}

\section{Jorge Renner Cardoso de Almeida ${ }^{1}$, Dina Michaela Kronhaus ${ }^{2}$, Etienne L. Sibille ${ }^{1}$, Scott A. Langenecker ${ }^{3}$, Amelia Versace ${ }^{1}$, Edmund James LaBarbara ${ }^{1}$ and Mary Louise Phillips ${ }^{1,4}$ *}

${ }^{1}$ Department of Psychiatry, University of Pittsburgh School of Medicine, Pittsburgh, PA, USA

2 St. Catharine's College and Computer Laboratory, University of Cambridge, Cambridge, UK

${ }^{3}$ University of Michigan Medical Center, Ann Arbor, MI, USA

${ }^{4}$ Department of Psychological Medicine, Cardiff University, Cardiff, UK

Edited by:

Alex Fornito, University of Melbourne, Australia

Reviewed by:

Christopher G. Davey, The University

of Melbourne, Australia

Udo Dannlowski, University of

Münster, Germany

*Correspondence:

Mary Louise Phillips, Director of

Functional Neuroimaging Program,

Western Psychiatric Institute and

Clinic, 3811 O'Hara Street, Pittsburgh,

PA 15213-2593, USA.

e-mail:phillipsm/@upmc.edu
Background: Pathophysiologic processes supporting abnormal emotion regulation in major depressive disorder (MDD) are poorly understood. We previously found abnormal inverse left-sided ventromedial prefrontal cortical-amygdala effective connectivity to happy faces in females with MDD. We aimed to replicate and expand this previous finding in an independent participant sample, using a more inclusive neural model, and a novel emotion processing paradigm. Methods: Nineteen individuals with MDD in depressed episode (12 females), and 19 healthy individuals, age, and gender matched, performed an implicit emotion processing and automatic attentional control paradigm to examine abnormalities in prefrontal cortical-amygdala neural circuitry during happy, angry, fearful, and sad face processing measured with functional magnetic resonance imaging in a 3-T scanner. Effective connectivity was estimated with dynamic causal modeling in a trinodal neural model including two anatomically defined prefrontal cortical regions, ventromedial prefrontal cortex, and subgenual cingulate cortex (sgACC), and the amygdala. Results: We replicated our previous finding of abnormal inverse left-sided top-down ventromedial prefrontal corticalamygdala connectivity to happy faces in females with $\operatorname{MDD}(p=0.04)$, and also showed a similar pattern of abnormal inverse left-sided sgACC-amygdala connectivity to these stimuli $(p=0.03)$. These findings were paralleled by abnormally reduced positive left-sided ventromedial prefrontal cortical-sgACC connectivity to happy faces in females with MDD $(p=0.008)$, and abnormally increased positive left-sided sgACC-amygdala connectivity to fearful faces in females, and all individuals, with $\operatorname{MDD}(p=0.008 ; p=0.003)$. Conclusion: Different patterns of abnormal prefrontal cortical-amygdala connectivity to happy and fearful stimuli might represent neural mechanisms for the excessive self-reproach and comorbid anxiety that characterize female MDD.

Keywords: major depressive disorder, effective connectivity, emotion regulation, dynamic causal modeling, amygdala, prefrontal cortex

\section{INTRODUCTION}

Major depressive disorder (MDD) is one of the most prevalent of all illnesses (Goodwin and Jamison, 2007). Elucidating neural system abnormalities reflecting pathophysiologic processes in MDD can help identify biological markers of the illness. The majority of neuroimaging studies in MDD focused on examination of activity within key neural regions supporting emotion processing and emotion regulation, including the amygdala and orbitomedial prefrontal cortex (OMPFC; Siegle et al., 2002, 2007; Anand et al., 2005; Dannlowski et al., 2007, 2009; Fales et al., 2008; Phillips et al., 2008a). Findings from these studies indicate abnormally increased amygdala activity to fear, happy, and neutral facial expressions (Sheline et al., 2001; Abler et al., 2007; Dannlowski et al., 2007; Fales et al., 2008), although others, no abnormalities in amygdala activity to emotional stimuli in individuals with
MDD (Lawrence et al., 2004; Surguladze et al., 2005; Fu et al., 2007; Dannlowski et al., 2008; Almeida et al., 2010b). Recent studies point to a valence specificity of amygdala hyperresponsiveness in MDD: while depressed patients were found to show stronger responsiveness to negative stimuli, less amygdala responsiveness to positive stimuli has been described (Suslow et al., 2010; Victor et al., 2010). The subgenual anterior cingulate cortex (sgACC, Brodmann Area, BA25) is one region of the OMPFC implicated in mood regulation and processing of negative self-referential information (Ongur et al., 2003; Phillips et al., 2008a; Price and Drevets, 2010), that has been the focus of an increasing number of neuroimaging studies in MDD. In MDD, volume in this region is reduced (Drevets, 2001; Bremner et al., 2002; Wagner et al., 2008), and resting blood flow in this region is increased (Mayberg et al., 1999; Jaracz and Rybakowski, 2002; Kito et al., 2008; Brockmann 
et al., 2009; Price and Drevets, 2010). Individuals with MDD who respond to antidepressant treatment have greater baseline activity and metabolism in sgACC (Saxena et al., 2003; Langenecker et al., 2007; Mulert et al., 2007; Korb et al., 2009; Keedwell et al., 2010), and the region has more recently been used as a biological target for deep brain stimulation for MDD (Gutman et al., 2009). Together, these studies highlight the roles of abnormal activity in amygdala and OMPFC in the pathophysiology of MDD.

Effective connectivity estimates are measures of the influence that different neural regions exert over others in a given neural system (Friston et al., 2003). These measures of function within a neural systems are potentially more consistent over time and sensitive than conventional neuroimaging measures of activity in different neural regions (Schuyler et al., 2010). Dynamic causal modeling (DCM) is an established method to estimate effective connectivity that has been validated with concomitant functional neuroimaging and electroencephalography (EEG), and intracerebral EEG in an animal model (David et al., 2008). The employment of effective connectivity in neuroimaging studies of psychiatric illness therefore has the potential to identify robust biological markers of MDD. Yet, few neuroimaging studies examined effective connectivity using DCM between neural regions of interest (ROI) in MDD (Schlösser et al., 2008; Almeida et al., 2009c). Some studies employed functional connectivity to examine correlation over time between regions in MDD. These studies reported decreased functional connectivity between subcortical regions (amygdala and ventral striatum) and prefrontal regions, including anterior cingulate gyrus (Anand et al., 2005; Chen et al., 2008; Matthews et al., 2008; Dannlowski et al., 2009; Heller et al., 2009; Frodl et al., 2010), consistent with the hypothesis of decreased prefrontal cortical regulation of subcortical limbic regions in MDD (Mayberg, 2003; Phillips et al., 2003; Anand et al., 2005; Price and Drevets, 2010). However, other studies showed increased connectivity within prefrontal areas (Hamilton et al., 2011), between prefrontal areas and thalamus (Greicius et al., 2007), and between prefrontal areas and affective, cognitive control and default mode networks in MDD (Sheline et al., 2010). Other studies showed normalization of abnormal anterior cingulate gyral-subcortical functional connectivity after pharmacological treatment in MDD (Anand et al., 2007; Chen et al., 2008). Replication of key neuroimaging findings is clearly essential to identify biological markers of MDD.

Using DCM, we recently demonstrated in individuals with MDD abnormal inverse left-sided effective connectivity to positive emotional stimuli (happy faces) between OMPFC and amygdala (Almeida et al., 2009c). Our finding was particularly evident in females with MDD (Cohen $d^{\prime}$ effect size of 0.97). There were two main limitations to this previous study, however. First, we did not directly compare females with males with MDD because the majority of our sample was female. The prevalence of MDD in females is almost double that in males (Goodwin and Jamison, 2007); yet, relationships between gender and neural system abnormalities in MDD remain poorly understood. Second, we included only one sub-region of the OMPFC, ventromedial prefrontal cortex (vmPFC, BA11).

In the present study, we therefore first aimed to examine the extent to which we could replicate our previous findings showing abnormal inverse left-sided vmPFC-amygdala connectivity to happy faces, especially in females with MDD. Secondly, we wished to extend our dynamic causal model to include sgACC as a third node to enhance our understanding of abnormal pathophysiologic mechanisms involved in MDD. We recruited a new group of male and female individuals with MDD, and employed a novel implicit emotion processing and automatic attention control of emotion paradigm. This paradigm allowed us to examine effective connectivity to positive and negative emotional faces (happy, anger, fear, and sad) in MDD and healthy individuals.

\section{MATERIALS AND METHODS PARTICIPANTS}

Nineteen healthy individuals ( 12 females and 7 males) and 19 individuals (12 females and 7 males) with SCID-verified (First et al., 1995 ) current MDD in depressed episode (no current or past psychosis) were recruited (Table 1 includes demographic and clinical data). These groups did not differ in age $[t(36)=0.7, p=0.5]$, or estimated premorbid intellectual ability [NART (Grober and Sliwinski, 1991), $t(36)=1.2, p=0.2$ ]. The Hamilton Rating Scale for Depression (Hamilton, 1960) score in individuals with MDD (HDRS-25-item, mean $=28.1, \mathrm{SD}=6.3$ ) was significantly greater than healthy individuals $[$ mean $=1.4, \mathrm{SD}=2.2 ; t(22.5)=17.5$, $p=0.0001]$. Most individuals with MDD were medicated $(78.9 \%$, see descriptive list in Table A1 in Appendix), most had lifetime history of comorbid anxiety disorder (63.2\%), while a proportion had lifetime history of comorbid substance disorder (26.3\%).

Exclusion criteria included history of head injury (from medical records and participant report), systemic medical illness, cognitive impairment (score $<24$ in the Mini-Mental State Examination, premorbid IQ estimate $<85$ using the National Adult Reading Test), Axis-II borderline personality disorder, and general exclusion criteria for MRI (presence/questionable history of metallic objects in the body, positive pregnancy test/self-reporting of pregnancy, and proneness to panicking in enclosed spaces). For healthy individuals, current alcohol and illicit substance abuse (determined by SCID-I, saliva, and urine screen) were further exclusion criteria.

The University of Pittsburgh Institutional Review Board approved the study protocol. All participants were right-handed. All participants were aware of the purpose of the study and gave written informed consent after explanation of the purpose of the research before participation in the study.

The participant population reflected the demographics of Pittsburgh and the surrounding area and/or the patient population of the University of Pittsburgh Medical Center (UPMC). Depressed participants were recruited from outpatient populations of the University of Pittsburgh Medical Center (UPMC), and through local advertising.

\section{MEDICATION LOAD}

A problem for all neuroimaging studies of psychiatric disorders is the potential confounding effect of psychotropic medication. We wished to examine the potential impact of psychotropic medication upon effective connectivity in MDD patients using an index of "medication load." This index reflects the number and dose of different medications for each individual: the greater the number and dose of the medication, the greater the medication load. This strategy has been employed in our previous neuroimaging studies (Hassel et al., 2008; Versace et al., 2008; Almeida et al., 2009a,c). 
Table 1 | Demographic, clinical variables, and behavior performance.

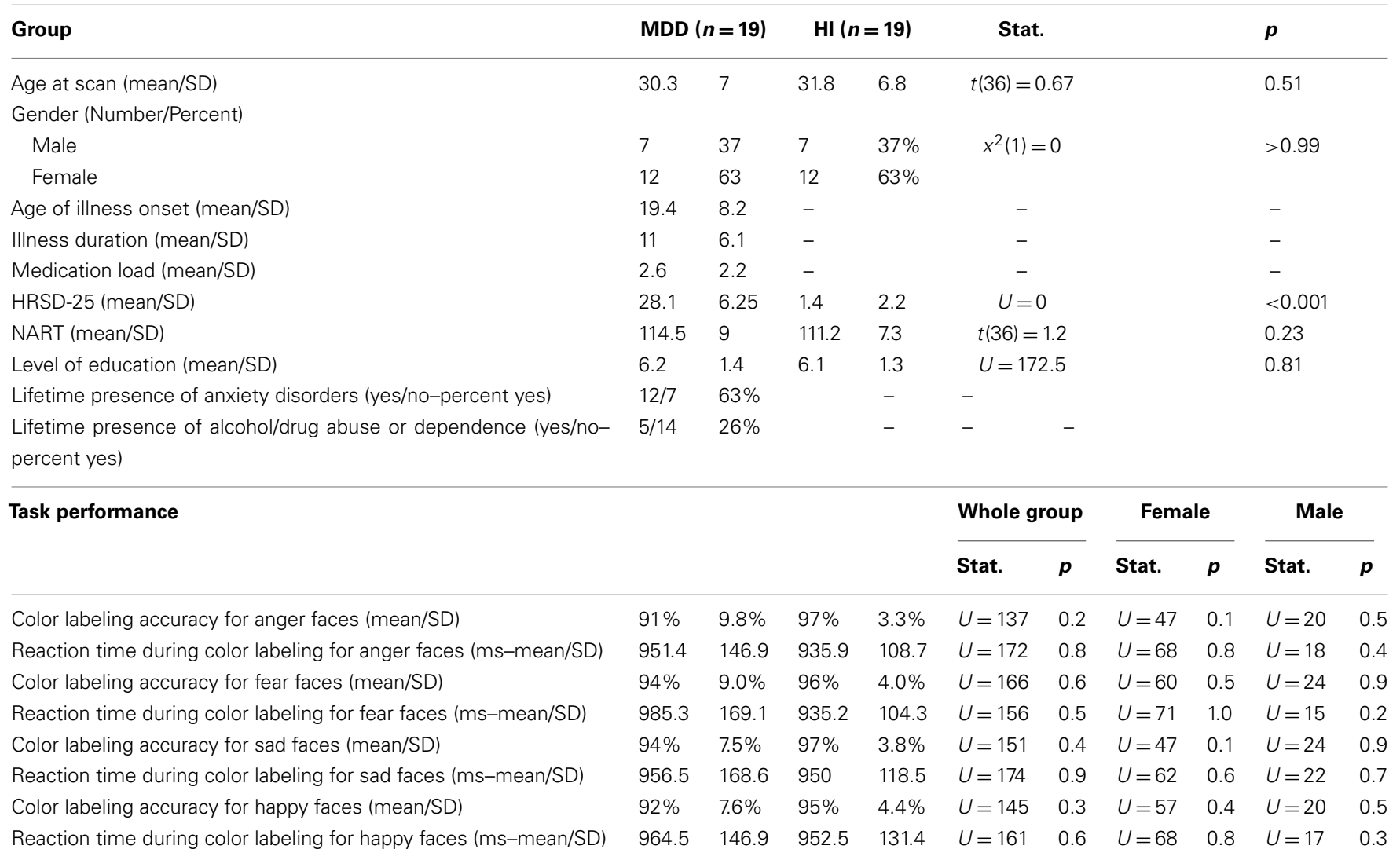

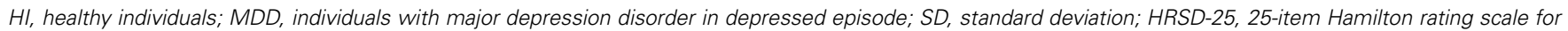
depression; Stat.: statistical test value; $p$ : $p$ value; $U=$ Mann-Whitney $U$ non-parametric test; ms, milliseconds; NART, national adult reading test.

To compute an index of medication load for each MDD participant, we first coded the dose of each antidepressant, moodstabilizer, antipsychotic, and anxiolytic medication as absent (0), low (1), or high (2). For antidepressants and mood-stabilizers, we converted each medication into low- or high-dose groupings using a previously employed approach (Sackeim, 2001). Individuals on levels 1 and 2 of these criteria were coded as low-dose, those with levels 3 and 4 as high-dose. We added a no-dose subtype for those not taking these medications. We converted antipsychotic doses into chlorpromazine dose equivalents, and coded as 0,1 , or 2 , for no medication, chlorpromazine equivalents dose equal or below, or above, the mean effective daily dose $\left(\mathrm{ED}_{50}\right)$ of chlorpromazine as defined by Davis and Chen (2004). Lorazepam dose was similarly coded as, 0,1 , or 2 , with reference to the midpoint of the Physician's Desk Reference-recommended daily dose range. We generated a composite measure of total medication load, reflecting dose, and variety of different medications taken, by summing all individual medication codes for each medication category for each individual MDD participant.

\section{DYNAMIC FACES TASK}

Participants completed a 12.5 -min emotional dynamic face processing task during the neuroimaging procedure. Participants were asked to use one of three fingers to press a button indicating the color of a semi-transparent foreground color flash (orange, turquoise, or yellow) that appeared during the mid 200-650 ms of a 1-s presentation of a dynamically changing background face (neutral to emotional; Figure 1). These emotional faces were considered task-irrelevant, as accurate task performance required participants to direct attention away from these faces and toward the color flash. The task therefore essentially measures implicit emotion processing and automatic attentional control of emotion. Faces from the NimStim stimulus set (Tottenham et al., 2009) were morphed in 5\% increments, from neutral (0\% emotion) to $100 \%$ emotion for four emotions: happy, sad, angry, and fearful. Morphed faces were made into $1 \mathrm{~s}$ movies progressing from 0 to $100 \%$ emotion. In control trials, movies comprised a simple shape (dark oval) superimposed on a light-gray oval, with similar structural characteristics to each face stimulus, which was subsequently morphed into a larger shape, approximating the movement shown by the morphed faces. There were three blocks for each of the above four types of emotion trial, with 12 stimuli per block, and six control (shape) blocks, with six stimuli per block. Emotional and control blocks were presented in a pseudorandomized order so that no two blocks of any condition were presented sequentially. Therefore, 36 stimuli per condition were presented in the task.

\section{fMRI DATA ANALYSES}

Neuroimaging data were collected using a 3-T Siemens Trio MRI scanner at the Magnetic Resonance Research Center in the University of Pittsburgh Medical Center (MRRC). Structural 3D axial MPRAGE images were acquired in the same session (TE: $3.29 \mathrm{~ms}$, 
TR: $2200 \mathrm{~ms}$, Flip angle $9^{\circ}, \mathrm{FOV}=256 \mathrm{~mm} \times 192 \mathrm{~mm}$, Slice thickness: $1 \mathrm{~mm}$, Matrix: $256 \times 256,192$ continuous slices). Bloodoxygen-level dependent (BOLD) images were then acquired with a gradient echo EPI sequence during 13 min covering 39 axial slices $(3.1 \mathrm{~mm}$ thick, TR/TE $=2000 / 28 \mathrm{~ms}, \mathrm{FOV}=205 \mathrm{~cm} \times 205 \mathrm{~cm}$, matrix $=64 \times 64$; Flip angle $90^{\circ}$ ).

Analyses were conducted in (SPM5; http://www.fil.ion.ucl.ac. $\mathrm{uk} / \mathrm{spm}$ ) with slice time correction, realignment, co-registration, normalized to MNI template, resampled to $2 \mathrm{~mm} \times 2 \mathrm{~mm} \times 2 \mathrm{~mm}$ voxels, and smoothed with a 8-mm FWHM Gaussian kernel.

\section{FUNCTIONAL SPECIALIZATION}

A first-level fixed-effect model was constructed with four emotions (anger, fear, sad, and happy) and the control condition entered as separate regressors in the design matrix, including movement parameters as nuisance variables and the canonical HRF model. The four emotion versus control contrasts were then entered into a second-level random-effects group analysis, that was conducted on the $t$-contrast images generated in the previous single-subject analyses in a two (group) by four (condition) repeated-measures analyses of variance (ANOVA). Bilateral ventromedial prefrontal cortex (BA11), bilateral subgenual anterior cingulate cortex (BA25), and bilateral amygdala ROIs included in our analyses were defined using the Wake Forest PickAtlas Talairach Daemon Brodmann Areas (Maldjian et al., 2003). To control for multiple statistical testing in the second-level randomeffect analysis, we maintained a cluster-level false positive detection rate at $p<0.05$ by using a voxel threshold of $p<0.05$ with a cluster $(k)$ extent empirically determined by Monte Carlo simulations implemented in AlphaSim, which accounted for spatial correlations between BOLD signal changes in neighboring voxels.

\section{FUNCTIONAL INTEGRATION: DYNAMIC CAUSAL MODELING ANALYSES}

Effective connectivity (termed "connectivity" from now on) was estimated using DCM (Friston et al., 2003; Mechelli et al., 2003) implemented in SPM8 software, as previously employed (Almeida et al., 2009b,c). The main goal was to replicate and extend our previous finding regarding abnormal inverse left-sided OMPFC (specifically the vmPFC and sgACC)-amygdala connectivity to happy faces. We therefore included these three anatomically defined regions in our dynamic causal model. DCM uses a neuronal model of distributed regional responses, and a validated

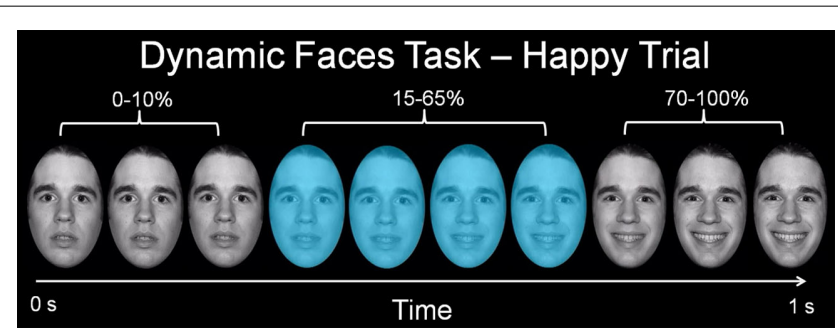

FIGURE 1 | Graphic representation of a single happy trial of our emotional dynamic faces task. Over a 1-s duration, the face changed from neutral (0\% emotion) to a happy, sad, angry, or fearful face (100\% emotion). Participants were asked to identify the color flash presented in mid dynamic change. biophysical model of hemodynamic signals, to model observed fMRI responses. The fitting or inversion of this model entails optimizing both the connection strength that couple different regions and the estimates of noise in each region. This optimization takes place in $\mathrm{E}$ and $\mathrm{M}$-steps respectively. Here the parameters correspond to both the region-specific hemodynamic parameters generating observed BOLD responses and the connectivity or coupling strengths among regions (Friston et al., 2003). In this study, we were primarily interested in endogenous connectivity among amygdala, vmPFC, and sgACC to emotional stimuli, and betweengroup differences in these connectivity measures. We therefore focused on examination of between-group differences in connectivity among the three regions to the four different emotion conditions. We generated a separate dynamic causal model for each emotion condition (see below). This individualized strategy allowed us, in effect, to examine the modulatory effect of emotion upon all endogenous connections in the model. We did not therefore use bilinear or modulatory terms in our dynamic causal model (to model changes in connectivity associated with change in condition).

The dynamic causal model for each emotion condition comprised bilateral amygdala, sgACC (BA25), and vmPFC (BA11), with reciprocal forward and backward ipsilateral connections among these three regions (Figure 2). The structure of this model was informed by understanding of the anatomical connections between these three regions from the large animal literature (Ongur et al., 2003; for review: Price and Drevets, 2010), that indicates bidirectional amygdala-vmPFC, amygdala-sgACC, and vmPFC-sgACC connections, with each region receiving sensory input from primary sensory processing cortices (Ongur et al., 2003; for review: Price and Drevets, 2010). Each emotion condition (happy, sad, angry, and fearful faces) was a separate input to each node in the model. This model therefore included in total 48 connectivity estimates per participants (three pairs of bidirectional connections in each hemisphere for each condition).

To account for individual differences, we extracted principal eigenvariates to summarize regional responses in anatomical

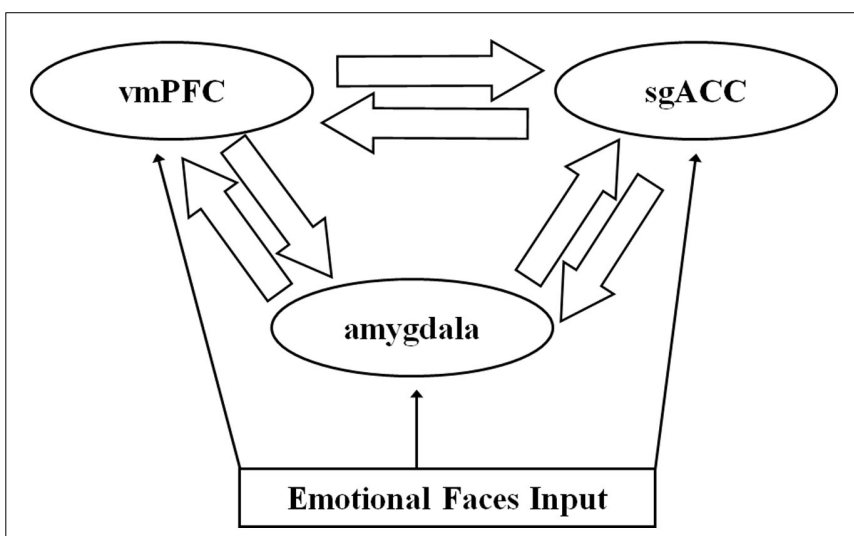

FIGURE 2 |The trinodal model including subgenual anterior cingulate cortex (sgACC), ventromedial prefrontal cortex (vmPFC), and amygdala and bidirectional connections among regions. Facial stimuli entered the model at each node. 
templates centered on the three regions above created with the Wake Forest University Pick Atlas (Maldjian et al., 2003). In DCM, the units of connections are per unit time and therefore correspond to rates: a strong connection means an influence that is expressed quickly or with a large rate-constant. A positive connection indicates that "high" activity in the "source" region is associated with an increase in activity in the "target" region, while a negative, or inverse, connection indicates that "high" activity in the "source" region is associated with a decrease in activity in the "target" region. The underlying model links rates of change in the target to the level of activity in the source (Friston et al., 2003).

\section{BETWEEN-GROUP DIFFERENCES IN CONNECTIVITY}

To assess between-group differences in connectivity we used a conventional summary statistic approach, where the within-subject estimates of coupling were passed to a second (between-subject) level for classical inference. Individual-specific estimates of connectivity were first entered into Predictive Analytics SoftWare (PASW, SPSS Inc.). We then used Mann-Whitney $U$-tests to compare groups on connectivity measures because data were not normally distributed.

Our primary analyses focused on our a priori connectivity measures: left sgACC-amygdala, and left vmPFC-amygdala to happy faces (statistical threshold set at $p<0.05$ ). We first compared all individuals with MDD versus healthy individuals, and then compared females with MDD versus healthy females, because we had specific a priori hypotheses about these connections in females with MDD based on our previous study (Almeida et al., 2009c). We also compared males with MDD relative to healthy males, although the smaller number of males made this comparison preliminary only.

In parallel analyses, we performed between-group comparisons on the remaining connectivity measures to all four emotion conditions (happy anger, fear, and sad). Here, we used the sequential goodness of fit (SGoF) metatest to correct for multiple test of between-group differences in the remaining 46 connectivity measures $(6$ pairs $\times 2$ hemispheres $\times 4$ emotional conditions, minus the above two a priori measures). The SGoF is an especially good method to correct for multiple tests in data sets where the sample size is not large, when the hypothesis to be tested may be weakly to moderately deviated from the null hypothesis, when there may be widespread effects through the family of tests, and when the number of tests is large (Carvajal-Rodriguez et al., 2009). The method is based on a comparison of the expected likely false positive error rate over all tests (computed from the per test significance level and the total number of tests performed), and the observed number of tests that meet the per test significance level. Contrary to other methods, the SGoF increases its statistical power with the number of tests, resolving in this way the trade-off between false positive finding (type I error) and statistical power (Carvajal-Rodriguez et al., 2009), and can show a statistical power up to two orders of magnitude higher than the Bonferroni method without increasing the false discovery rate (FDR). The SGoF is therefore an appropriate analytic strategy for multitest correction when working with high-dimensional biological data.

\section{EXPLORATORY ANALYSES}

In exploratory analyses, we compared healthy males with females, and males with females with MDD on connectivity measures that showed significant between-group differences. Connectivity measures showing significant abnormalities were also explored for possible relationships, using Spearman rank correlation tests and Mann-Whitney $U$-tests as appropriate, with: age, age of illness onset, illness duration, depression severity (measured using the HRSD-25), medication load (see Appendix), taking versus not taking individual psychotropic medication classes, and lifetime history of comorbid anxiety and/or substance disorder. Here, in individuals with MDD, we used a statistical threshold of $p=0.05 / 10=0.005$, to control for the 10 separate tests for each connectivity measure. For healthy individuals, relationships between connectivity measures and age and subthreshold depression symptom severity using the HRSD-25 were examined using Spearman rank correlation tests and a statistical threshold of $p=0.05 / 2=0.025$, to control for the two tests for each connectivity measure.

\section{RESULTS}

\section{TASK PERFORMANCE}

Task performance accuracy was high: 91\% face color labeling accuracy for individuals with MDD, and 94\% face color labeling accuracy for healthy individuals. There were no significant between-group differences in all individuals, females, or males in accuracy or reaction time, for any emotion condition (Table 1).

\section{FUNCTIONAL SPECIALIZATION ON vmPFC, sgACC, AND AMYGDALA ROIs}

We found no significant main effect of group or interaction; however, we found a significant positive effect of condition in all ROI bilaterally. Thus, revealing that the individuals recruited these regions to perform the task (Table 2).

\section{DYNAMIC CAUSAL MODELING}

\section{Primary analyses: between-group differences in left}

vmPFC-amygdala and sgACC-amygdala connectivity to happy faces Comparison of all individuals with MDD and healthy individuals did not reveal any significant differences in left vmPFC-amygdala or sgACC-amygdala connectivity to happy faces. Comparison of females with MDD relative to healthy females replicated our previous finding of significantly greater inverse left-sided vmPFCamygdala connectivity to happy faces, and extended this to also show significantly greater inverse left-sided sgACC-amygdala connectivity in females with MDD relative to healthy females ( $p=0.04$ and $p=0.02$, respectively). Males with MDD did not differ significantly from healthy males in either of these connectivity measures (Table 3; Figure 3).

\section{Parallel analysis: remaining connectivity measures}

For all individuals with MDD versus all healthy individuals, five pairwise between-group comparisons met the per test significance threshold of $p=0.05$ (Table 4). One of these comparisons survived SGoF correction: all individuals with MDD relative to healthy individuals showed significantly greater positive leftsided sgACC-amygdala connectivity $(p=0.003)$ to fearful faces (Table 3; Figure 4). 
Table 2 | Effective connectivity estimates in a priori and parallel analysis to happy, angry, sad, and fearful faces.

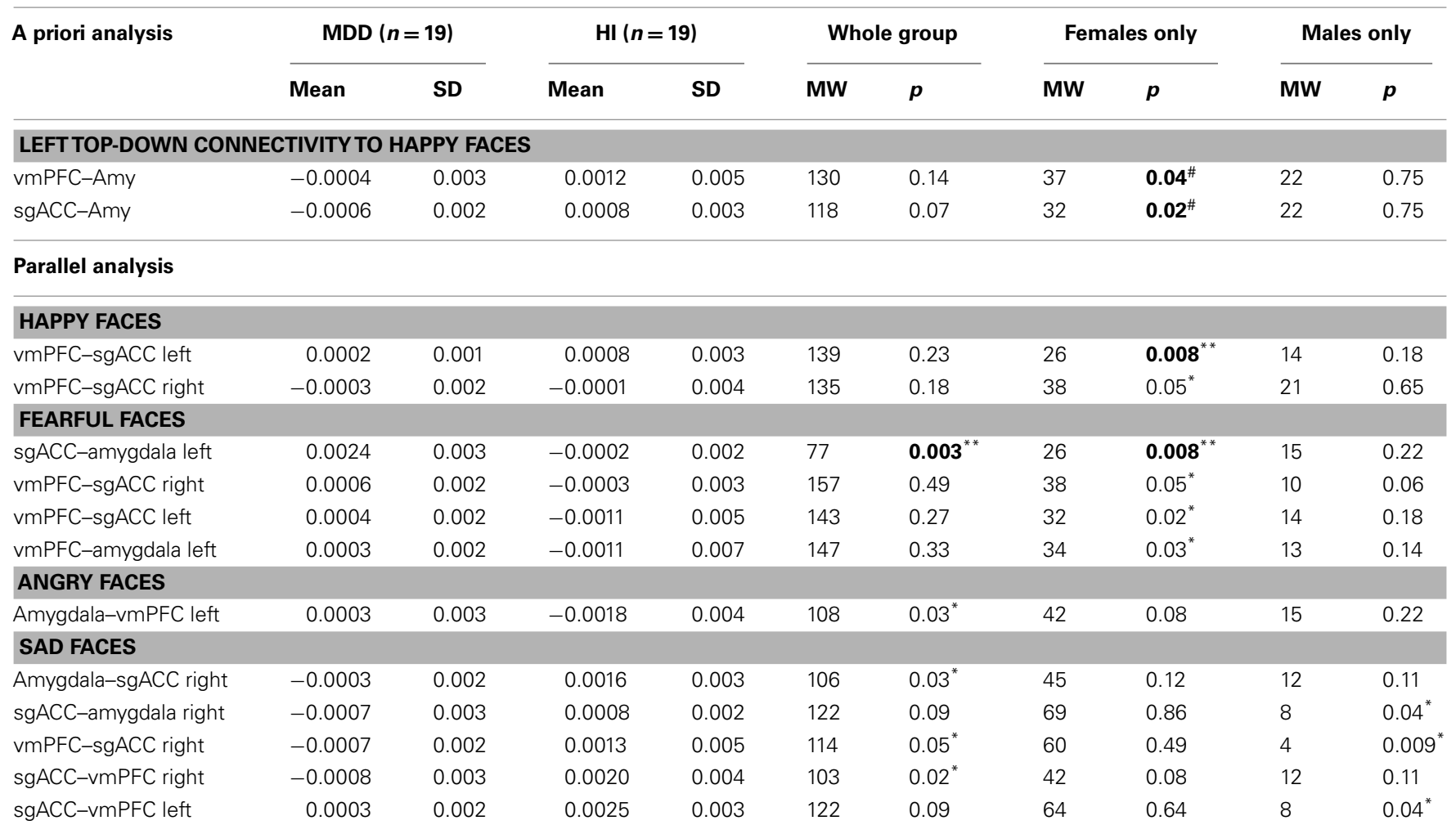

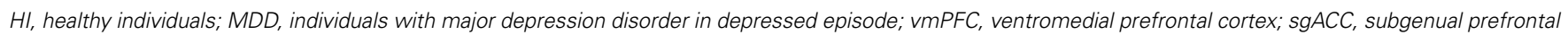

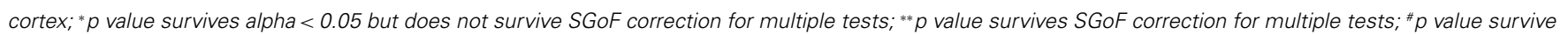
a priori alpha threshold of $p<0.05 ; S D$, standard deviation; MW, Mann-Whitney U non-parametric test; SGoF, sequential goodness of fit.

Table 3 | Amygdala, vmPFC, and sgACC activity during the dynamic faces task.

\begin{tabular}{|c|c|c|c|c|c|c|c|c|}
\hline \multirow[t]{2}{*}{ Region } & \multirow[t]{2}{*}{ Side } & \multicolumn{3}{|c|}{ Mini coordinators } & \multirow[t]{2}{*}{$K$} & \multirow[t]{2}{*}{$T$} & \multirow[t]{2}{*}{$Z$} & \multirow[t]{2}{*}{$P$} \\
\hline & & $x$ & $Y$ & $Z$ & & & & \\
\hline \multirow[t]{2}{*}{ vmPFC (BA11) } & $\mathrm{R}$ & 4 & 52 & -14 & 28 & 2.61 & 2.57 & $0.05^{* *}$ \\
\hline & $\mathrm{L}$ & -4 & 54 & -12 & 11 & 2.23 & 2.21 & $0.013^{*}$ \\
\hline sgACC (BA25) & $\mathrm{R}$ & 2 & 8 & -14 & 7 & 2.18 & 2.14 & $0.016^{*}$ \\
\hline \multirow[t]{2}{*}{ Amygdala } & $\mathrm{R}$ & 20 & -4 & -16 & 114 & 3.72 & 3.63 & $0.024^{* *}$ \\
\hline & $\mathrm{L}$ & -20 & -6 & -16 & 113 & 2.89 & 2.84 & $0.049^{* *}$ \\
\hline
\end{tabular}

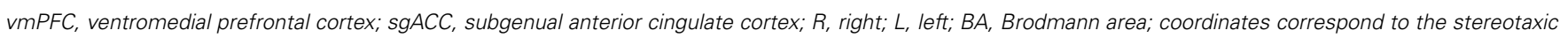
array of Montreal Neurologic Institute; $k$ : cluster size; * alphasim correction; ** false discovery rate correction.

For females with MDD versus healthy females, six pairwise between-group comparisons met the per test significance threshold of $p=0.05$ (Table 4). Two of these comparisons survived SGoF correction. 1. to happy faces: significantly reduced positive left-sided vmPFC-sgACC connectivity $(p=0.008)$ in females with MDD relative to healthy females. 2 . to fearful faces: significantly greater positive left-sided sgACC-amygdala connectivity $(p=0.008)$, where females with MDD showed a positive connectivity, healthy females showed an inverse connectivity (Table 3; Figure 4).
For males with MDD relative to healthy males, three pairwise between-group comparisons met the threshold of $p=0.05$, but none survived SGoF correction (Tables 3 and 4).

\section{Within-group gender comparison}

In healthy individuals, the left-sided vmPFC-sgACC connectivity to happy faces that survived the SGoF correction for betweengroup differences in connectivity in female participants showed a significant effect of gender. Here, healthy females had positive, and healthy males, inverse connectivity between these regions 

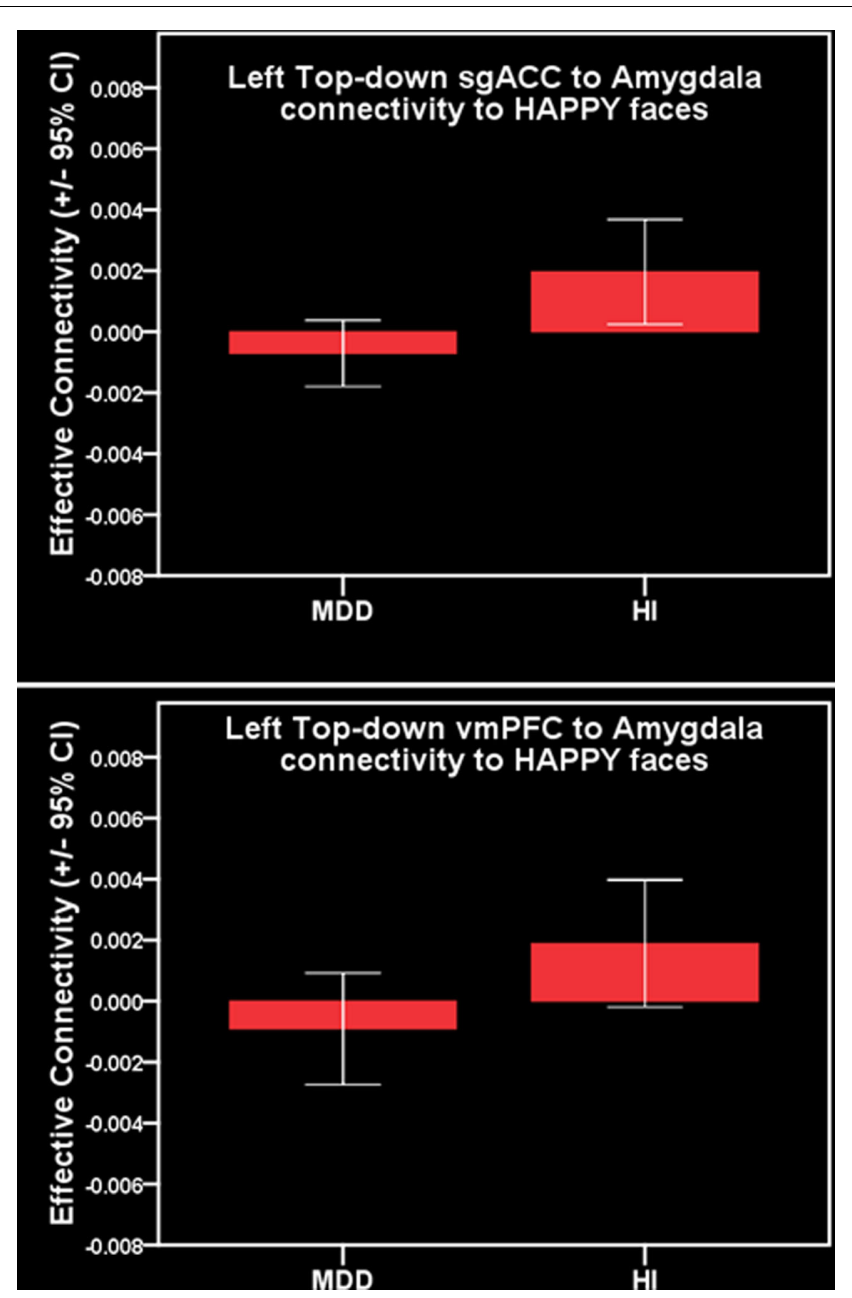

FIGURE 3 | Primary a priori analysis. Top: inverse left-sided vmPFC-amygdala connectivity to happy faces in females with MDD Bottom: inverse left-sided sgACC-amygdala connectivity to happy faces in females with MDD.

( $p=0.022$ ). In individuals with MDD, there were no significant effects of gender on any of the connectivity measures that survived the SGoF correction for examination of between-group differences in connectivity.

\section{Exploratory relationships between abnormal connectivity and age, task performance, and illness history}

In individuals with MDD, there were no significant relationships between any clinical, demographic, or task performance measure and connectivity measures showing between-group differences in connectivity using the Bonferroni-corrected threshold of $p \leq 0.005$ (Table 5). In healthy individuals, however, there was a significant negative correlation between sub-syndromal depressive symptoms, and left sgACC-amygdala connectivity to happy faces $(p=0.01)$. Here, greater sub-syndromal depressive symptom severity was associated with reduced left-sided sgACC-amygdala connectivity to happy faces (Table 6).

\section{DISCUSSION}

The goal of the present study was to identify neuroimaging measures of recurrent MDD in females by examining the extent to which we could replicate in an independent sample, of females with MDD, and extend to a trinodal model, our previous finding of abnormal inverse left-sided OMPFC-amygdala connectivity to positive emotional stimuli (happy faces). In a priori hypothesis testing, we replicated our previous finding of abnormal inverse left-sided vmPFC-amygdala connectivity, and also showed abnormal inverse left-sided sgACC-amygdala connectivity, to happy faces in females (but not males) with MDD. Parallel analyses across all other remaining connectivity measures in our trinodal dynamic causal model revealed abnormally reduced positive leftsided vmPFC-sgACC connectivity, to happy faces, and abnormal positive left-sided sgACC-amygdala connectivity to fearful faces in females with MDD. Our present and previous findings highlight the fact that gender is an important factor to be considered in neuroimaging studies of MDD, and that abnormal left-sided OMPFC-amygdala connectivity during processing of both happy and fearful stimuli may be specific to female MDD.

Females experience depression at a rate twice that of males (Kendler et al., 2002; McGrath et al., 2009). Females are also more likely than males to have atypical depression, characterized by transient mood reactivity to actual or potential positive events, increase in appetite, hypersomnia, interpersonal rejection sensitivity and/or comorbid anxiety (APA, 2000), and are more likely to describe interpersonal stress and excessive self-reproach (Hammen, 2003; Smith et al., 2008). Interpretation of the abnormal patterns of connectivity between vmPFC, sgACC, and amygdala to happy and fearful faces in females with MDD in the context of these clinical features of female MDD can be guided by previous studies that elucidated the roles of the vmPFC and sgACC in emotion regulation. Both vmPFC and sgACC are part of the medial network within OMPFC implicated in emotion and visceromotor regulation (Ongur et al., 2003; Price and Drevets, 2010) and have strong reciprocal connections with the amygdala (Ongur et al., 2003; Price and Drevets, 2010). Human neuroimaging studies implicate both bilateral sgACC (Williams et al., 2006) and vmPFC (Van Veen and Carter, 2002; Luks et al., 2007) in fear extinction, an example of automatic behavioral control of emotion (Phillips et al., 2008b). The left vmPFC is also implicated in aversive conditioning (Gottfried and Dolan, 2004), while left/bilateral sgACC are implicated in the generation of sad mood (Mayberg, 1999), depression (Mayberg, 2005), and elevated monitoring of internal states in individuals with attachment avoidant personality styles (Gillath et al., 2005). The pattern of abnormal inverse or reduced positive connectivity between sgACC, vmPFC, and amygdala to happy faces may therefore reflect abnormal inhibition, or reduced functional integration, in this circuitry during positive emotion processing, that may lead to abnormally increased regulation of emotional response to, and reduced representation of the positive emotional value of, positive emotional stimuli in female MDD. This in turn may be associated with only transient positive mood reactivity to positive events and excessive self-reproach observed in female MDD. By contrast, the abnormally elevated left-sided positive sgACC-amygdala connectivity to fearful faces in females 
Table 4 | Effective connectivity estimates to happy, fearful, sad, and angry faces.

\begin{tabular}{|c|c|c|c|c|c|c|c|c|c|c|}
\hline & \multicolumn{2}{|c|}{$\operatorname{MDD}(n=19)$} & \multicolumn{2}{|c|}{$\mathrm{HI}(n=19)$} & \multicolumn{2}{|c|}{ Whole group } & \multicolumn{2}{|c|}{ Females only } & \multicolumn{2}{|c|}{ males only } \\
\hline & Mean & SD & Mean & SD & MW & $p$ & MW & $p$ & MW & $p$ \\
\hline Amygdala-sgACC left & 0.0010 & 0.002 & 0.0000 & 0.003 & 153 & 0.42 & 66 & 0.73 & 19 & 0.48 \\
\hline Amygdala-sgACC right & 0.0013 & 0.003 & -0.0002 & 0.003 & 141 & 0.25 & 58 & 0.42 & 19 & 0.48 \\
\hline Amygdala-vmPFC left & 0.0021 & 0.006 & -0.0003 & 0.003 & 148 & 0.34 & 51 & 0.23 & 22 & 0.75 \\
\hline sgACC-Amy left & -0.0006 & 0.002 & 0.0008 & 0.003 & 118 & 0.07 & 32 & $0.02^{*}$ & 22 & 0.75 \\
\hline sgACC-amygdala right & 0.0003 & 0.002 & -0.0004 & 0.003 & 172 & 0.80 & 60 & 0.49 & 23 & 0.85 \\
\hline sgACC-vmPFC left & 0.0001 & 0.002 & 0.0020 & 0.005 & 147 & 0.33 & 51 & 0.23 & 22 & 0.75 \\
\hline sgACC-vmPFC right & 0.0000 & 0.002 & 0.0010 & 0.003 & 144 & 0.29 & 50 & 0.20 & 24 & 0.95 \\
\hline vmPFC-Amy left & -0.0004 & 0.003 & 0.0012 & 0.005 & 130 & 0.14 & 37 & $0.04^{\#}$ & 22 & 0.75 \\
\hline vmPFC-amygdala right & -0.0005 & 0.003 & -0.0004 & 0.005 & 158 & 0.51 & 66 & 0.73 & 19 & 0.48 \\
\hline Amygdala-sgACC left & 0.0003 & 0.002 & -0.0005 & 0.003 & 120 & 0.08 & 46 & 0.13 & 23 & 0.85 \\
\hline Amygdala-sgACC right & -0.0002 & 0.003 & 0.0000 & 0.004 & 148 & 0.34 & 39 & 0.06 & 14 & 0.18 \\
\hline Amygdala-vmPFC left & 0.0003 & 0.003 & -0.0018 & 0.004 & 108 & $0.03^{*}$ & 42 & 0.08 & 15 & 0.22 \\
\hline Amygdala-vmPFC right & -0.0001 & 0.002 & -0.0002 & 0.004 & 140 & 0.24 & 65 & 0.69 & 12 & 0.11 \\
\hline sgACC-amygdala left & -0.0003 & 0.003 & 0.0012 & 0.005 & 158 & 0.51 & 51 & 0.23 & 22 & 0.75 \\
\hline sgACC-amygdala right & -0.0011 & 0.006 & -0.0001 & 0.004 & 167 & 0.69 & 65 & 0.69 & 16 & 0.28 \\
\hline sgACC-vmPFC left & -0.0006 & 0.003 & -0.0013 & 0.007 & 175 & 0.87 & 71 & 0.95 & 24 & 0.95 \\
\hline sgACC-vmPFC right & -0.0003 & 0.002 & -0.0010 & 0.006 & 158 & 0.51 & 48 & 0.17 & 18 & 0.41 \\
\hline vmPFC-amygdala left & -0.0005 & 0.002 & 0.0009 & 0.005 & 162 & 0.59 & 71 & 0.95 & 17 & 0.34 \\
\hline vmPFC-amygdala right & -0.0015 & 0.004 & -0.0001 & 0.005 & 180 & 0.99 & 55 & 0.33 & 15 & 0.22 \\
\hline vmPFC-sgACC left & 0.0011 & 0.003 & 0.0011 & 0.005 & 167 & 0.69 & 67 & 0.77 & 18 & 0.41 \\
\hline sgACC-amygdala right & 0.0004 & 0.003 & -0.0003 & 0.003 & 152 & 0.41 & 44 & 0.11 & 20 & 0.57 \\
\hline sgACC-vmPFC left & 0.0002 & 0.005 & -0.0011 & 0.004 & 140 & 0.24 & 56 & 0.36 & 19 & 0.48 \\
\hline sgACC-vmPFC right & 0.0004 & 0.005 & -0.0007 & 0.004 & 158 & 0.51 & 63 & 0.60 & 22 & 0.75 \\
\hline vmPFC-amygdala left & 0.0003 & 0.002 & -0.0011 & 0.007 & 147 & 0.33 & 34 & $0.03^{*}$ & 13 & 0.14 \\
\hline vmPFC-amygdala right & 0.0003 & 0.002 & -0.0012 & 0.005 & 134 & 0.17 & 40 & 0.06 & 21 & 0.65 \\
\hline vmPFC-sgACC left & 0.0004 & 0.002 & -0.0011 & 0.005 & 143 & 0.27 & 32 & $0.02^{*}$ & 14 & 0.18 \\
\hline vmPFC-sgACC right & 0.0006 & 0.002 & -0.0003 & 0.003 & 157 & 0.49 & 38 & $0.05^{*}$ & 10 & 0.06 \\
\hline \multicolumn{11}{|l|}{ SAD FACES } \\
\hline Amygdala-sgACC left & -0.0008 & 0.004 & 0.0012 & 0.004 & 158 & 0.51 & 66 & 0.73 & 12 & 0.11 \\
\hline Amygdala-sgACC right & -0.0003 & 0.002 & 0.0016 & 0.003 & 106 & $0.03^{*}$ & 45 & 0.12 & 12 & 0.11 \\
\hline Amygdala-vmPFC left & 0.0006 & 0.003 & 0.0018 & 0.004 & 179 & 0.97 & 66 & 0.73 & 22 & 0.75 \\
\hline Amygdala-vmPFC right & 0.0006 & 0.003 & 0.0013 & 0.003 & 167 & 0.69 & 66 & 0.73 & 21 & 0.65 \\
\hline sgACC-amygdala left & -0.0008 & 0.002 & 0.0013 & 0.003 & 129 & 0.13 & 57 & 0.39 & 13 & 0.14 \\
\hline sgACC-amygdala right & -0.0007 & 0.003 & 0.0008 & 0.002 & 122 & 0.09 & 69 & 0.86 & 8 & $0.04^{*}$ \\
\hline sgACC-vmPFC left & 0.0003 & 0.002 & 0.0025 & 0.003 & 122 & 0.09 & 64 & 0.64 & 8 & $0.04^{*}$ \\
\hline sgACC-vmPFC right & -0.0008 & 0.003 & 0.0020 & 0.004 & 103 & $0.02^{*}$ & 42 & 0.08 & 12 & 0.11 \\
\hline vmPFC-amygdala left & -0.0002 & 0.004 & 0.0010 & 0.004 & 139 & 0.23 & 58 & 0.42 & 16 & 0.28 \\
\hline vmPFC-amygdala right & -0.0005 & 0.003 & 0.0013 & 0.005 & 144 & 0.29 & 60 & 0.49 & 17 & 0.34 \\
\hline
\end{tabular}


Table 4 | Continued

\begin{tabular}{|c|c|c|c|c|c|c|c|c|c|c|}
\hline & \multicolumn{2}{|c|}{$\operatorname{MDD}(n=19)$} & \multicolumn{2}{|c|}{$\mathrm{HI}(n=19)$} & \multicolumn{2}{|c|}{ Whole group } & \multicolumn{2}{|c|}{ Females only } & \multicolumn{2}{|c|}{ males only } \\
\hline & Mean & SD & Mean & SD & MW & $p$ & MW & $p$ & MW & $p$ \\
\hline vmPFC-sgACC right & -0.0007 & 0.002 & 0.0013 & 0.005 & 114 & $0.05^{*}$ & 60 & 0.49 & 4 & $0.009^{*}$ \\
\hline
\end{tabular}

HI, healthy individuals; MDD, individuals with major depression disorder in depressed episode; vmPFC, ventromedial prefrontal cortex; sgACC, subgenual prefrontal cortex; " $p$ value survives alpha < 0.05 but does not survive SGoF correction for multiple tests; ${ }^{* *} p$ value survives SGoF correction for multiple tests; ${ }^{\circ} p$ value survive a priori alpha threshold of $p<0.05 ; S D$, standard deviation; MW, Mann-Whitney U non-parametric test; SGoF, sequential goodness of fit.

with MDD may represent a neural mechanism for the abnormally increased representation of threat depicted by fearful faces, which in turn may be related to the comorbid anxiety and interpersonal stress associated with female MDD.

Interpretation of the abnormal patterns of sgACC-amygdala connectivity to happy and fearful faces in females with MDD can also be guided by previous neuroimaging studies that focused on the sgACC in MDD. Previous studies provide increasing evidence that differential patterns of sgACC activity to negative versus positive emotional stimuli may moderate and mediate response to different antidepressant treatments in MDD. Previous findings indicate, for example, that greater levels of activity in individuals with MDD in the sgACC to negative emotional stimuli may predict subsequent successful response to antidepressant treatment (Saxena et al., 2003; Langenecker et al., 2007; Mulert et al., 2007; Korb et al., 2009; Keedwell et al., 2010). Other findings show that successful response to antidepressant treatments is paralleled by decreased activity in sgACC to negative emotional stimuli (Anand et al., 2007; Fu et al., 2007; Chen et al., 2008; Keedwell et al., 2010), but by greater activity in this region to positive emotional stimuli (Fu et al., 2007; Keedwell et al., 2010). These findings therefore suggest that differential patterns of sgACC activity to negative versus positive emotional stimuli may reflect pathophysiologic processes of MDD. Little is yet known, however, regarding the extent to which differential patterns of abnormal connectivity between the sgACC and amygdala to positive versus negative emotional stimuli may reflect different pathophysiologic processes associated with female versus male MDD, or may moderate and mediate treatment response in the illness in females and males.

No previous neuroimaging studies directly compared females and males with MDD during emotion processing tasks. Previous studies that focused only on females with MDD indicate abnormal OMPFC and amygdala activity to emotional stimuli in females with MDD versus healthy females. For example, these previous studies showed abnormally increased bilateral amygdala activity to negative emotional stimuli, and abnormally increased left sgACC activity to positive emotional stimuli (Abler et al., 2007), although no difference in amygdala or OMPFC activity during a cognitive control of emotion task between females with high severity depression symptoms compared to females with low depression symptoms (Beevers et al., 2010). Females with MDD recovered from depression were reported to show greater right amygdala and reduced left ACC activity to parental criticism stimuli, and reduced right ACC activity to parental praise and neutral stimuli compared to healthy females (Hooley et al., 2009). Previous studies

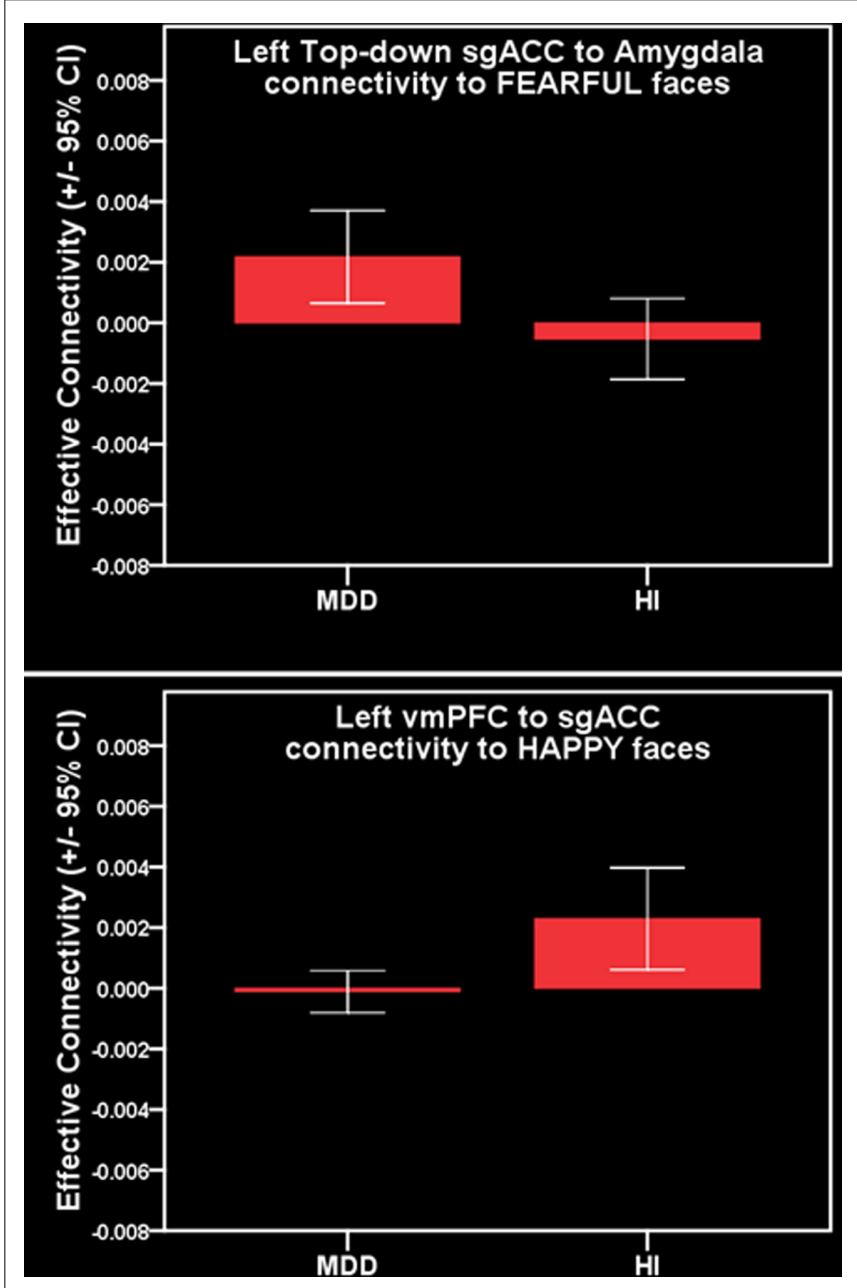

FIGURE 4 | Parallel analysis. Top: greater positive left-sided sgACC-amygdala connectivity to fearful faces in females with MDD. Bottom: reduced positive left-sided vmPFC-sgACC connectivity to happy faces in females with MDD.

also indicate greater activity in left ACC and right lateral prefrontal cortex to positive emotional stimuli in females with MDD versus healthy females (Mitterschiffthaler et al., 2003). Another study showed greater left ACC and left dorsolateral prefrontal cortical activity (Wagner et al., 2006) and greater effective connectivity between left dorsal and rostral ACC during performance of the 
Table 5 | Relation between effective connectivity, clinical, demographic, and experiment performance variables in individuals with MDD.

\begin{tabular}{|c|c|c|c|c|c|c|c|c|c|c|}
\hline & \multicolumn{6}{|c|}{ Happy faces } & \multirow{2}{*}{\multicolumn{4}{|c|}{\begin{tabular}{l}
\multicolumn{1}{c}{ Fearful faces } \\
Left-sided \\
sgACC-Amy EC
\end{tabular}}} \\
\hline & \multirow{2}{*}{\multicolumn{2}{|c|}{$\begin{array}{l}\text { Left-sided } \\
\text { sgACC-Amy EC } \\
\text { Females only; } \\
\text { MDD ( } n=12)\end{array}$}} & \multirow{2}{*}{\multicolumn{2}{|c|}{$\begin{array}{l}\text { Left-sided } \\
\text { vmPFC-Amy EC } \\
\text { Females only; } \\
\text { MDD ( } n=12)\end{array}$}} & \multirow{2}{*}{\multicolumn{2}{|c|}{$\begin{array}{l}\text { Left-sided } \\
\text { vmPFC-sgACC EC } \\
\text { Females only; } \\
\text { MDD ( } n=12)\end{array}$}} & & & & \\
\hline & & & & & & & \multicolumn{2}{|c|}{$\begin{array}{l}\text { Whole group; } \\
\text { MDD ( } n=19 \text { ) }\end{array}$} & \multicolumn{2}{|c|}{$\begin{array}{l}\text { Females only; } \\
\text { MDD ( } n=12)\end{array}$} \\
\hline & Rho & $p$ Value & Rho & $p$ Value & Rho & $p$ Value & Rho & $p$ Value & Rho & $p$ Value \\
\hline Age at scan & 0.08 & 0.80 & 0.24 & 0.46 & 0.13 & 0.68 & -0.12 & 0.63 & 0.13 & 0.70 \\
\hline Age of illness onset & -0.18 & 0.58 & 0.35 & 0.26 & 0.41 & 0.18 & 0.00 & 0.99 & -0.05 & 0.87 \\
\hline Illness duration & 0.15 & 0.65 & -0.24 & 0.46 & -0.37 & 0.24 & 0.06 & 0.81 & 0.17 & 0.59 \\
\hline Medication load & 0.23 & 0.47 & 0.06 & 0.86 & -0.21 & 0.51 & -0.49 & $0.03^{*}$ & -0.22 & 0.50 \\
\hline HRSD-25 & 0.30 & 0.34 & 0.13 & 0.69 & -0.04 & 0.91 & -0.17 & 0.48 & -0.36 & 0.24 \\
\hline Accuracy emotional face & -0.23 & 0.47 & 0.13 & 0.69 & -0.25 & 0.43 & -0.24 & 0.32 & -0.09 & 0.79 \\
\hline \multirow[t]{2}{*}{ Reaction time emotional face } & 0.14 & 0.66 & -0.04 & 0.90 & 0.57 & 0.06 & -0.08 & 0.75 & 0.13 & 0.68 \\
\hline & MW & $p$ Value & MW & $p$ Value & MW & $p$ Value & MW & $p$ Value & MW & $p$ Value \\
\hline Mood stabilizators (on/off) & 0 & 0.11 & 1 & 0.19 & 4 & 0.66 & 12 & 0.18 & 2 & 0.31 \\
\hline Anti psychotic medications (on/off) & 6 & 0.39 & 4 & 0.20 & 10 & 1.00 & 19 & 0.58 & 5 & 0.28 \\
\hline Anti depressants (on/off) & 12 & 0.78 & 10 & 0.52 & 8 & 0.30 & 13 & 0.09 & 9 & 0.41 \\
\hline Benzodiazepines (on/off) & 4 & 0.08 & 12 & 0.78 & 12 & 0.78 & 28 & 0.24 & 12 & 0.78 \\
\hline Lifetime presence of alcohol/drugs & 13 & 0.93 & 11 & 0.64 & 12 & 0.78 & 13 & $0.04^{*}$ & 6 & 0.17 \\
\hline \multicolumn{11}{|l|}{ Abuse or dependence (yes/no) } \\
\hline $\begin{array}{l}\text { Lifetime presence of anxiety disor- } \\
\text { der (yes/no) }\end{array}$ & 16 & 0.81 & 8 & 0.12 & 13 & 0.46 & 16 & $0.03^{*}$ & 5 & $0.04^{*}$ \\
\hline
\end{tabular}

MDD, individuals with major depression disorder in depressed episode; MW, Mann-Whitney U; Rho: Spearman rank correlation; HRSD-25: 25-item Hamilton rating scale for depression; vmPFC, ventromedial prefrontal cortex; sgACC, subgenual prefrontal cortex; ${ }^{p}$ does not survive multiple correction; EC, effective connectivity.

Table 6 | Relation between effective connectivity, clinical, demographic, and experiment performance variables in HI.

\begin{tabular}{|c|c|c|c|c|c|c|c|c|c|c|}
\hline & \multicolumn{6}{|c|}{ Happy faces } & \multicolumn{4}{|c|}{ Fearful faces } \\
\hline & \multirow{2}{*}{\multicolumn{2}{|c|}{$\begin{array}{l}\text { Left-sided } \\
\text { sgACC-Amy EC } \\
\text { Females only } \\
\text { HI }(n=12)\end{array}$}} & \multirow{2}{*}{\multicolumn{2}{|c|}{$\begin{array}{l}\text { Left-sided } \\
\text { vmPFC-Amy EC } \\
\text { Females only } \\
\text { HI }(n=12)\end{array}$}} & \multirow{2}{*}{\multicolumn{2}{|c|}{$\begin{array}{l}\text { Left-sided } \\
\text { vmPFC-sgACC EC } \\
\text { Females only } \\
\text { HI }(n=12)\end{array}$}} & \multicolumn{4}{|c|}{$\begin{array}{l}\text { Left-sided } \\
\text { sgACC-Amy EC }\end{array}$} \\
\hline & & & & & & & \multicolumn{2}{|c|}{$\begin{array}{l}\text { Whole group } \\
\text { HI }(n=19)\end{array}$} & \multicolumn{2}{|c|}{$\begin{array}{l}\text { Females only } \\
\text { HI (n=12) }\end{array}$} \\
\hline & Rho & $p$ Value & Rho & $p$ Value & Rho & $p$ Value & Rho & $p$ Value & Rho & $p$ Value \\
\hline Age at scan & -0.59 & $0.04^{*}$ & -0.40 & 0.20 & -0.43 & 0.16 & -0.35 & 0.15 & -0.59 & $0.04^{*}$ \\
\hline HRSD-25 & -0.69 & $0.01^{* *}$ & -0.48 & 0.11 & -0.26 & 0.42 & -0.06 & 0.82 & -0.32 & 0.32 \\
\hline Accuracy emotional face & -0.19 & 0.55 & -0.27 & 0.40 & 0.00 & 1.00 & -0.45 & $0.05^{*}$ & -0.34 & 0.28 \\
\hline Reaction time Emotional face & -0.50 & 0.10 & -0.37 & 0.24 & -0.31 & 0.32 & 0.24 & 0.33 & 0.31 & 0.33 \\
\hline
\end{tabular}

HI, healthy individuals; Rho, Spearman rank correlation; HRSD-25, 25-item Hamilton rating scale for depression; vmPFC, ventromedial prefrontal cortex; sgACC, subgenual prefrontal cortex; * $p$ does not survive multiple correction; ** $p$ value survives multiple correction; EC, effective connectivity.

incongruent condition of the Stroop task in females with MDD relative to healthy females (Schlösser et al., 2008). Together with our present findings, these findings therefore suggest abnormal functional integration between, predominantly, left-sided OMPFC and bilateral amygdala to positive and negative emotional stimuli, and also during attentional tasks, in females with MDD. Further studies are clearly needed to elucidate pathophysiologic processes that may differ between male and female MDD.
A popular theory emphasizes the role of the left hemisphere in processing approach-related emotional stimuli (Davidson et al., 1990; Sutton and Davidson, 1997). Cognitive neuroscience accounts of face processing, however, highlight the role of the left hemisphere as critical in "detail-oriented" processing, whereas the right hemisphere may support "gestalt" processing (Sergent and Bindra, 1981; Rossion et al., 2000). Our findings therefore suggest that the left-lateralized pattern of abnormal 
connectivity in females with MDD may reflect not only abnormal processing of approach-related emotional stimuli, but also the employment of detail-oriented left-hemisphere-centered strategies during implicit processing of the emotional faces, and automatic regulation of response to these stimuli, to a greater extent than other groups. This should be the focus of future studies.

Examination of gender differences in connectivity measures that were abnormal in individuals with MDD revealed significantly greater inverse left-sided vmPFC-sgACC connectivity to happy faces in healthy males relative to healthy females, such that healthy males showed a pattern of connectivity between these regions that was more similar to that shown by females with MDD than that shown by healthy females. These findings suggest that one pathophysiologic process in female MDD is to render the functional integrity within left-sided vmPFC-sgACC circuitry during positive emotion processing similar to that shown by healthy males, but this requires further study. Interestingly, in healthy individuals, more severe sub-syndromal depression was associated with significantly reduced left-sided sgACC-amygdala connectivity to happy faces, a connectivity pattern that resembled females with MDD.

While a limitation of our study was the small number of males with MDD, our main focus was to examine OMPFC-amygdala connectivity to positive and negative emotional stimuli in females with MDD. Another limitation was the recruitment of medicated individuals with MDD, although our findings indicate normalizing rather than confounding effects of such medication upon one of our connectivity measures, providing a valuable insight into putative neural mechanisms that may mediate antidepressant treatment response in MDD. Further replications of our current and previous findings are clearly needed in future studies. Future studies can determine whether the observed group differences represent state or trait markers of depression by including individuals who have recovered from depression in analyses. Future studies can also aim to examine the extent to which adolescents and adults

\section{REFERENCES}

Abler, B., Erk, S., Herwig, U., and Walter, H. (2007). Anticipation of aversive stimuli activates extended amygdala in unipolar depression. J. Psychiatr. Res. 41, 511-522.

Almeida, J. R., Akkal, D., Hassel, S., Travis, M. J., Banihashemi, L., Kerr, N., Kupfer, D. J., and Phillips, M. L. (2009a). Reduced gray matter volume in ventral prefrontal cortex but not amygdala in bipolar disorder: significant effects of gender and trait anxiety. Psychiatry Res. 171, 54-68.

Almeida, J. R., Mechelli, A., Hassel, S., Versace, A., Kupfer, D. J., and Phillips, M. L. (2009b). Abnormally increased effective connectivity between parahippocampal gyrus and ventromedial prefrontal regions during emotion labeling in bipolar disorder. Psychiatry Res. 174, 195-201.
Almeida, J. R., Versace, A., Mechelli, A. Hassel, S., Quevedo, K., Kupfer, D. J., and Phillips, M. L. (2009c). Abnormal amygdala-prefrontal effective connectivity to happy faces differentiates bipolar from major depression. Biol. Psychiatry 66, 451-459.

Almeida, J. R. C., Phillips, M. L., Cerqueira, C. T., Zilberman, M., Lobo, D., Henna, E., Tavares, H., Amaro, E., Gorenstein, C., Gentil, V., and Busatto, G. F. (2010a). Neural activity changes to emotional stimuli in healthy individuals under chronic use of clomipramine. J. Psychopharmacol. (Oxford) 24, 1165-1174.

Almeida, J. R. C., Versace, A., Hassel, S., Kupfer, D. J., and Phillips, M. L. (2010b). Elevated amygdala activity to sad facial expressions: a state marker of bipolar but not unipolar depression. Biol. Psychiatry 67, 414-421. at potential future risk of mood disorders show these patterns of abnormal connectivity between vmPFC and amygdala during emotion processing paradigms. Furthermore, given promising findings from activation studies in MDD, showing that baseline activity in frontocingulate areas may predict future treatment response in MDD (Almeida et al., 2010a; Pizzagalli, 2011), future studies can also aim to examine the extent to which measures of abnormal connectivity may also predict treatment response in MDD.

In conclusion, we replicated our previous finding of abnormal inverse left-sided OMPFC-amygdala connectivity to positive emotional stimuli in an independent group of females with MDD using a novel implicit emotion processing paradigm. Our present findings show a more extensive pattern of abnormal left-sided connectivity among sgACC, vmPFC, and amygdala to happy and fearful faces in females, but not males, with MDD. The differential pattern of abnormal left-sided OMPFC-amygdala connectivity to happy and fearful faces may therefore represent a neural mechanism for female MDD, which is characterized by comorbid anxiety and high levels of interpersonal stress. Our findings highlight the utility of effective connectivity studies in identifying gender-specific markers of MDD that may help identify potential biological targets for novel, personalized treatments for individuals with MDD.

\section{ACKNOWLEDGMENTS}

All work was carried out within the Department of Psychiatry, University of Pittsburgh, neuroimaging data was collected at the Magnetic Resonance Research Center (MRRC), University of Pittsburgh. We thank Dr. Fernando Boada and his staff for their help acquiring neuroimaging data. Dr. Phillips reports having support from R01 MH076971-01. Dr.Versace reports support from a NARSAD young investigator award, Dr. Almeida, Dr. Kronhaus, Mr. LaBarbara, reported no biomedical financial interests or potential conflicts of interest.
Anand, A., Li, Y., Wang, Y., Gardner, K., and Lowe, M. J. (2007). Reciprocal effects of antidepressant treatment on activity and connectivity of the mood regulating circuit: an FMRI study. J. Neuropsychiatry Clin. Neurosci. 19, 274-282.

Anand, A., Li, Y., Wang, Y., Wu, J., Gao, S., Bukhari, L., Mathews, V. P., Kalnin, A., and Lowe, M. J. (2005). Activity and connectivity of brain mood regulating circuit in depression: a functional magnetic resonance study. Biol. Psychiatry 57, 1079-1088.

APA. (2000). Diagnostic and Statistical Manual of Mental Disorders: DSM$I V$-TR. Washington, DC: American Psychiatric Association.

Beevers, C. G., Clasen, P., Stice, E. and Schnyer, D. (2010). Depression symptoms and cognitive control of emotion cues: a functional magnetic resonance imaging study. Neuroscience 167, 97-103.
Bremner, J. D., Vythilingam, M., Vermetten, E., Nazeer, A., Adil, J., Khan, S., Staib, L. H., and Charney, D. S. (2002). Reduced volume of orbitofrontal cortex in major depression. Biol. Psychiatry 51, 273-279.

Brockmann, H., Zobel, A., Joe, A. Biermann, K., Scheef, L., Schuhmacher, A., Von Widdern, O., Metten, M., Biersack, H. J., Maier, W., and Boecker, H. (2009). The value of HMPAO SPECT in predicting treatment response to citalopram in patients with major depression. Psychiatry Res. 173, 107-112.

Carvajal-Rodriguez, A., De UnaAlvarez, J., and Rolan-Alvarez, E. (2009). A new multitest correction (SGoF) that increases its statistical power when increasing the number of tests. BMC Bioinformatics 10, 209. doi:10.1186/1471-2105-10-209 
Chen, C. H., Suckling, J., Ooi, C., Fu, C. H., Williams, S. C., Walsh, N. D., Mitterschiffthaler, M. T., Pich, E. M., and Bullmore, E. (2008). Functional coupling of the amygdala in depressed patients treated with antidepressant medication. Neuropsychopharmacology 33, 1909-1918.

Dannlowski, U., Ohrmann, P., Bauer, J., Deckert, J., Hohoff, C., Kugel, H., Arolt, V., Heindel, W., Kersting, A., Baune, B. T., and Suslow, T. (2008). 5-HTTLPR biases amygdala activity in response to masked facial expressions in major depression. Neuropsychopharmacology 33, 418-424.

Dannlowski, U., Ohrmann, P., Bauer, J., Kugel, H., Arolt, V., Heindel, W., and Suslow, T. (2007). Amygdala reactivity predicts automatic negative evaluations for facial emotions. Psychiatry Res. 154, 13-20.

Dannlowski, U., Ohrmann, P., Konrad, C., Domschke, K., Bauer, J., Kugel, H., Hohoff, C., Schoning, S., Kersting, A., Baune, B. T., Mortensen, L. S., Arolt, V., Zwitserlood, P., Deckert, J., Heindel, W., and Suslow, T. (2009). Reduced amygdala-prefrontal coupling in major depression: association with MAOA genotype and illness severity. Int. J. Neuropsychopharmacol. 12, 11-22.

David, O., Guillemain, I., Saillet, S., Reyt, S., Deransart, C., Segebarth, C., and Depaulis, A. (2008). Identifying neural drivers with functional MRI: an electrophysiological validation. PLoS Biol. 6, e315. doi:10.1371/journal.pbio.0060315

Davidson, R. J., Ekman, P., Saron, C. D., Senulis, J. A., and Friesen, W. V. (1990). Approach-withdrawal and cerebral asymmetry: emotional expression and brain physiology. I. J. Pers. Soc. Psychol. 58, 330-341.

Davis, J. M., and Chen, N. (2004). Dose response and dose equivalence of antipsychotics. J. Clin. Psychopharmacol. 24, 192-208.

Drevets, W. C. (2001). Neuroimaging and neuropathological studies of depression: implications for the cognitive-emotional features of mood disorders. Curr. Opin. Neurobiol. 11, 240-249.

Fales, C. L., Barch, D. M., Rundle, M. M., Mintun, M. A., Snyder, A. Z., Cohen, J. D., Mathews, J., and Sheline, Y. I. (2008). Altered emotional interference processing in affective and cognitive-control brain circuitry in major depression. Biol. Psychiatry 63, 377-384.

First, M. B., Spitzer, R. L., Gibbon, M., and Willians, J. B. W. (1995). Structured Clinical Interview for DSMIV Axis I Disorders (SCID-, version
2.0). New York: Biometric research Department, New York State Psychiatric Institute.

Friston, K. J., Harrison, L., and Penny, W. (2003). Dynamic causal modelling. Neuroimage 19, 1273-1302.

Frodl, T., Bokde, A. L. W., Scheuerecker, J., Lisiecka, D., Schoepf, V., Hampel, H., Möller, H.-J., Brückmann, H., Wiesmann, M., and Meisenzahl, E. (2010). Functional connectivity bias of the orbitofrontal cortex in drug-free patients with major depression. Biol. Psychiatry 67, 161-167.

Fu, C. H., Williams, S. C., Brammer, M. J., Suckling, J., Kim, J., Cleare, A. J., Walsh, N. D., Mitterschiffthaler, M. T., Andrew, C. M., Pich, E. M., and Bullmore, E. T. (2007). Neural responses to happy facial expressions in major depression following antidepressant treatment. Am. J. Psychiatry 164, 599-607.

Gillath, O., Bunge, S. A., Shaver, P. R., Wendelken, C., and Mikulincer, M. (2005). Attachment-style differences in the ability to suppress negative thoughts: exploring the neural correlates. Neuroimage 28, 835-847.

Goodwin, F. K., and Jamison, K. R. (2007). Manic-Depressive Illness: Bipolar Disorders and Recurrent Depression. New York, NY: Oxford University Press.

Gottfried, J. A., and Dolan, R. J. (2004). Human orbitofrontal cortex mediates extinction learning while accessing conditioned representations of value. Nat. Neurosci. 7, 1144-1152.

Greicius, M. D., Flores, B. H., Menon, V., Glover, G. H., Solvason, H. B., Kenna, H., Reiss, A. L., and Schatzberg, A. F. (2007). Resting-state functional connectivity in major depression: abnormally increased contributions from subgenual cingulate cortex and thalamus. Biol. Psychiatry 62, 429-437.

Grober, E., and Sliwinski, M. (1991). Development and validation of a model for estimating premorbid verbal intelligence in the elderly. J. Clin. Exp. Neuropsychol. 13, 933-949.

Gutman, D. A., Holtzheimer, P. E., Behrens, T. E. J., Johansen-Berg, H., and Mayberg, H. S. (2009). A tractography analysis of two deep brain stimulation white matter targets for depression. Biol. Psychiatry 65, 276-282.

Hamilton, J. P., Chen, G., Thomason, M. E., Schwartz, M. E., and Gotlib, I. H. (2011). Investigating neural primacy in major depressive disorder: multivariate Granger causality analysis of resting-state fMRI time-series data. Mol. Psychiatry 16, 763-772.
Hamilton, M. (1960). A rating scale for depression. J. Neurol. Neurosurg. Psychiatry 23, 56-62.

Hammen, C. (2003). Interpersonal stress and depression in women. $J$. Affect. Disord. 74, 49-57.

Hassel, S., Almeida, J. R. C., Kerr N., Nau, S., Ladouceur, C. D., Fissell, K., Kupfer, D. J., and Phillips, M. L. (2008). Elevated striatal and decreased dorsolateral prefrontal cortical activity in response to emotional stimuli in euthymic bipolar disorder: no associations with psychotropic medication load. Bipolar Disord. 10, 916-927.

Heller, A. S., Johnstone, T., Shackman, A. J., Light, S. N., Peterson, M. J., Kolden, G. G., Kalin, N. H., and Davidson, R. J. (2009). Reduced capacity to sustain positive emotion in major depression reflects diminished maintenance of fronto-striatal brain activation. Proc. Natl. Acad. Sci. U.S.A. 106, 22445-22450.

Hooley, J. M., Gruber, S. A., Parker, H. A., Guillaumot, J., Rogowska, J., and Yurgelun-Todd, D. A. (2009). Cortico-limbic response to personally challenging emotional stimuli after complete recovery from depression. Psychiatry Res. 172, 83-91.

Jaracz, J., and Rybakowski, J. (2002). Studies of cerebral blood flow in metabolism in depression using positron emission tomography (PET). Psychiatr. Pol. 36, 617-628.

Keedwell, P. A., Drapier, D., Surguladze, S., Giampietro, V., Brammer, M., and Phillips, M. (2010). Subgenual cingulate and visual cortex responses to sad faces predict clinical outcome during antidepressant treatment for depression. J. Affect. Disord. 120, 120-125.

Kendler, K. S., Gardner, C. O., and Prescott, C. A. (2002). Toward a comprehensive developmental model for major depression in women. Am. J. Psychiatry 159, 1133-1145.

Kito, S., Fujita, K., and Koga, Y (2008). Changes in regional cerebral blood flow after repetitive transcranial magnetic stimulation of the left dorsolateral prefrontal cortex in treatment-resistant depression. J. Neuropsychiatry Clin. Neurosci. 20, 74-80.

Korb, A. S., Hunter, A. M., Cook, I. A. and Leuchter, A. F. (2009). Rostral anterior cingulate cortex theta current density and response to antidepressants and placebo in major depression. Clin. Neurophysiol. 120, 1313-1319.

Langenecker, S. A., Kennedy, S. E., Guidotti, L. M., Briceno, E. M., Own, L. S., Hooven, T., Young, E. A., Akil,
H., Noll, D. C., and Zubieta, J.-K. (2007). Frontal and limbic activation during inhibitory control predicts treatment response in major depressive disorder. Biol. Psychiatry 62, 1272-1280.

Lawrence, N. S., Williams, A. M., Surguladze, S., Giampietro, V., Brammer, M. J., Andrew, C., Frangou, S., Ecker, C., and Phillips, M. L. (2004) Subcortical and ventral prefrontal cortical neural responses to facial expressions distinguish patients with bipolar disorder and major depression. Biol. Psychiatry 55, 578-587.

Luks, T. L., Simpson, G. V., Dale, C. L., and Hough, M. G. (2007). Preparatory allocation of attention and adjustments in conflict processing. Neuroimage 35, 949-958.

Maldjian, J. A., Laurienti, P. J., Kraft, R. A., and Burdette, J. H. (2003). An automated method for neuroanatomic and cytoarchitectonic atlas-based interrogation of fMRI data sets. Neuroimage 19, 1233-1239.

Matthews, S. C., Strigo, I. A., Simmons, A. N., Yang, T. T., and Paulus, M. P. (2008). Decreased functional coupling of the amygdala and supragenual cingulate is related to increased depression in unmedicated individuals with current major depressive disorder. J. Affect. Disord. 111, 13-20.

Mayberg, H. S. (1999). Reciprocal limbic-cortical function and negative mood: converging PET findings in depression and normal sadness. Am. J. Psychiatry 156, 675-682.

Mayberg, H. S. (2003). Modulating dysfunctional limbic-cortical circuits in depression: towards development of brain-based algorithms for diagnosis and optimised treatment. $\mathrm{Br}$. Med. Bull. 65, 193-207.

Mayberg, H. S. (2005). Deep brain stimulation for treatment-resistant depression. Neuron 45, 651-660.

Mayberg, H. S., Liotti, M., Brannan, S. K., Mcginnis, S., Mahurin, R. K., Jerabek, P. A., Silva, J. A. Tekell, J. L., Martin, C. C., Lancaster, J. L., and Fox, P. T. (1999). Reciprocal limbic-cortical function and negative mood: converging PET findings in depression and normal sadness. Am. J. Psychiatry 156, 675-682.

McGrath, E., Keita, G. P., Strickland, B. R., and Russo, N. F. (2009). Women and Depression: Risk Factors and Treatment Issues: Final Report of the American Psychological Association's National Task Force on Women and Depression. Washington, DC: American Psychological Association. 
Mechelli, A., Price, C. J., Noppeney, U., and Friston, K. J. (2003). A dynamic causal modeling study on category effects: bottom-up or topdown mediation? J. Cogn. Neurosci. 15, 925-934.

Mitterschiffthaler, M. T., Kumari, V., Malhi, G. S., Brown, R. G., Giampietro, V. P., Brammer, M. J., Suckling, J., Poon, L., Simmons, A., Andrew, C., and Sharma, T. (2003). Neural response to pleasant stimuli in anhedonia: an fMRI study. Neuroreport 14, 177-182.

Mulert, C., Juckel, G., Brunnmeier, M., Karch, S., Leicht, G., Mergl, R., Moller, H. J., Hegerl, U., and Pogarell, O. (2007). Rostral anterior cingulate cortex activity in the theta band predicts response to antidepressive medication. Clin. EEG Neurosci. 38, 78-81.

Ongur, D., Ferry, A. T., and Price, J. L. (2003). Architectonic subdivision of the human orbital and medial prefrontal cortex. J. Comp. Neurol. 460, 425-449.

Phillips, M. L., Drevets, W. C., Rauch, S. L., and Lane, R. (2003). Neurobiology of emotion perception II: implications for major psychiatric disorders. Biol. Psychiatry 54, 515-528.

Phillips, M. L., Ladouceur, C. D., and Drevets, W. C. (2008a). A neural model of voluntary and automatic emotion regulation: implications for understanding the pathophysiology and neurodevelopment of bipolar disorder. Mol. Psychiatry 13, 829, 833-857.

Phillips, M. L., Ladouceur, C. D., and Drevets, W. C. (2008b). A neural model of voluntary and automatic emotion regulation: implications for understanding the pathophysiology and neurodevelopment of bipolar disorder. Mol. Psychiatry 13, 833-857.

Pizzagalli, D. A. (2011). Frontocingulate dysfunction in depression: toward biomarkers of treatment response. Neuropsychopharmacology 36, 183-206.

Price, J. L., and Drevets, W. C. (2010). Neurocircuitry of mood disorders. Neuropsychopharmacology 35, 192-216.

Rossion, B., Dricot, L., Devolder, A., Bodart, J.-M., Crommelinck, M., Gelder, B. D., and Zoontjes, R.
(2000). Hemispheric asymmetries for whole-based and part-based face processing in the human fusiform gyrus. J. Cogn. Neurosci. 12, 793-802.

Sackeim, H. A. (2001). The definition and meaning of treatmentresistant depression. J. Clin. Psychiatry 62(Suppl. 16), 10-17.

Saxena, S., Brody, A. L., Ho, M. L., Zohrabi, N., Maidment, K. M., and Baxter, L. R. Jr. (2003). Differential brain metabolic predictors of response to paroxetine in obsessivecompulsive disorder versus major depression. Am. J. Psychiatry 160, 522-532.

Schlösser, R. G. M., Wagner, G., Koch, K., Dahnke, R., Reichenbach, J. R., and Sauer, H. (2008). Frontocingulate effective connectivity in major depression: a study with fMRI and dynamic causal modeling. $\mathrm{Neu}$ roimage 43, 645-655.

Schuyler, B., Ollinger, J. M., Oakes, T. R., Johnstone, T., and Davidson, R J. (2010). Dynamic causal modeling applied to fMRI data shows high reliability. Neuroimage 49, 603-611.

Sergent, J., and Bindra, D. (1981). Differential hemispheric processing of faces: methodological considerations and reinterpretation. Psychol. Bull. 89, 541-554.

Sheline, Y. I., Barch, D. M., Donnelly, J. M., Ollinger, J. M., Snyder, A. Z., and Mintun, M. A. (2001). Increased amygdala response to masked emotional faces in depressed subjects resolves with antidepressant treatment: an fMRI study. Biol. Psychiatry 50, 651-658.

Sheline, Y. I., Price, J. L., Yan, Z., and Mintun, M. A. (2010). Restingstate functional MRI in depression unmasks increased connectivity between networks via the dorsal nexus. Proc. Natl. Acad. Sci. U.S.A. 107, 11020-11025.

Siegle, G. J., Steinhauer, S. R., Thase, M. E., Stenger, V. A., and Carter, C. S. (2002). Can't shake that feeling: event-related fMRI assessment of sustained amygdala activity in response to emotional information in depressed individuals. Biol. Psychiatry 51, 693-707.

Siegle, G. J., Thompson, W., Carter, C. S., Steinhauer, S. R., and Thase, M. E. (2007). Increased amygdala and decreased dorsolateral prefrontal BOLD responses in unipolar depression: related and independent features. Biol. Psychiatry 61, 198-209.

Smith, D. J., Kyle, S., Forty, L., Cooper, C., Walters, J., Russell, E., Caesar, S., Farmer, A., Mcguffin, P., Jones, I., Jones, L., and Craddock, N. (2008). Differences in depressive symptom profile between males and females. J. Affect. Disord. 108, 279-284.

Surguladze, S., Brammer, M. J., Keedwell, P., Giampietro, V., Young, A. W. Travis, M. J., Williams, S. C. R., and Phillips, M. L. (2005). A differential pattern of neural response toward sad versus happy facial expressions in major depressive disorder. Biol. Psychiatry 57, 201.

Suslow, T., Konrad, C., Kugel, H., Rumstadt, D., Zwitserlood, P., Schöning, S., Ohrmann, P., Bauer, J., Pyka, M. Kersting, A., Arolt, V., Heindel, W. and Dannlowski, U. (2010). Automatic mood-congruent amygdala responses to masked facial expressions in major depression. Biol. Psychiatry 67, 155-160.

Sutton, S. K., and Davidson, R. J. (1997). Prefrontal brain asymmetry: a biological substrate of the behavioral approach and inhibition systems. Psychol. Sci. 8, 204-210.

Tottenham, N., Tanaka, J. W., Leon, A. C., Mccarry, T., Nurse, M., Hare, T. A., Marcus, D. J., Westerlund, A., Casey, B. J., and Nelson, C. (2009). The NimStim set of facial expressions: judgments from untrained research participants. Psychiatry Res. $168,242-249$.

Van Veen, V., and Carter, C. S. (2002). The timing of action-monitoring processes in the anterior cingulate cortex. J. Cogn. Neurosci. 14 593-602.

Versace, A., Almeida, J. R., Hassel, S., Walsh, N. D., Novelli, M., Klein, C. R., Kupfer, D. J., and Phillips, M. L. (2008). Elevated left and reduced right orbitomedial prefrontal fractional anisotropy in adults with bipolar disorder revealed by tract-based spatial statistics. Arch. Gen. Psychiatry 65, 1041-1052.

Victor, T. A., Furey, M. L., Fromm, S. J., Ohman, A., and Drevets, W. C. (2010). Relationship between amygdala responses to masked faces and mood state and treatment in major depressive disorder. Arch. Gen. Psychiatry 67, 1128-1138.

Wagner, G., Koch, K., Schachtzabel, C., Reichenbach, J. R., Sauer, H., and Schlosser Md, R. G. (2008). Enhanced rostral anterior cingulate cortex activation during cognitive control is related to orbitofrontal volume reduction in unipolar depression. J. Psychiatry Neurosci. 33, 199-208.

Wagner, G., Sinsel, E., Sobanski, T., Kohler, S., Marinou, V., Mentzel, H. J., Sauer, H., and Schlosser, R. G. (2006). Cortical inefficiency in patients with unipolar depression: an event-related FMRI study with the Stroop task. Biol. Psychiatry 59, 958-965.

Williams, L. M., Das, P., Liddell, B. J., Kemp, A. H., Rennie, C. J., and Gordon, E. (2006). Mode of functional connectivity in amygdala pathways dissociates level of awareness for signals of fear. J. Neurosci. 26, 9264-9271.

Conflict of Interest Statement: The authors declare that the research was conducted in the absence of any commercial or financial relationships that could be construed as a potential conflict of interest.

Received: 30 August 2011; accepted: 18 November 2011; published online: 08 December 2011.

Citation: Almeida JRC, Kronhaus DM, Sibille EL, Langenecker SA, Versace A, LaBarbara EJ and Phillips ML (2011) Abnormal left-sided orbitomedial prefrontal cortical-amygdala connectivity during happy and fear face processing: a potential neural mechanism of female MDD. Front. Psychiatry 2:69. doi: 10.3389/fpsyt.2011.00069

This article was submitted to Frontiers in Neuropsychiatric Imaging and Stimulation, a specialty of Frontiers in Psychiatry. Copyright (c) 2011 Almeida, Kronhaus, Sibille, Langenecker, Versace, LaBarbara and Phillips. This is an open-access article distributed under the terms of the Creative Commons Attribution Non Commercial License, which permits noncommercial use, distribution, and reproduction in other forums, provided the original authors and source are credited. 


\section{APPENDIX}

Table A1 | Descriptive list of medication for each individual with major depressive disorder.

\begin{tabular}{|c|c|c|c|c|c|}
\hline \multicolumn{3}{|l|}{ Subject } & \multicolumn{3}{|l|}{ Drug name } \\
\hline 2 & N/A & & & & \\
\hline 4 & Fluvoxamine & Clonazepam & Risperidona & & \\
\hline 5 & Fluoxetine & Clonazepam & & & \\
\hline 6 & Fluoxetine & & & & \\
\hline 8 & N/A & & & & \\
\hline 9 & Lithium & Trazodone & Clonazepam & & \\
\hline 10 & Paroxetine & Temazepam & Chlorpromazine & Gabapentin & Lorazepam \\
\hline 11 & Venlafaxine & Trazodone & & & \\
\hline 12 & Lorazepam & Desipramine & Lamotrigine & Mirtazapine & \\
\hline 17 & Alprazolam & Citalopram & & & \\
\hline 18 & N/A & & & & \\
\hline 19 & Aripiprazole & Zolpidem & Venlafaxine & & \\
\hline
\end{tabular}

